\title{
A FREQUENCY DOMAIN APPROACH FOR NON-COHERENT TIMING CHIP-LEVEL SYNCHRONIZATION FOR CDMA SYSTEMS
}

\author{
Cristiano Panazio* \\ Escola Politécnica of the University of São Paulo (EPUSP) \\ Departament of Telecommunications and Control (PTC) \\ Av. Prof. Luciano Gualberto, tr. 3, \\ Cid. Universitária, São Paulo SP, \\ Brazil, CEP 05508-900
}

\begin{abstract}
In this paper, we propose a simple computational chip-level non-coherent timing synchronization technique operating in the frequency domain. The proposed technique almost attains the modified Cramér-Rao bound when the system operates with small roll-off values of the transmitter/receiver filters. Furthermore, the frequency domain processing allows to easily mitigate the noxious effect of strong narrowband interference. The proposed method is discussed and analyzed. We provide some analytical and numerical results to show its effectiveness.
\end{abstract}

\section{INTRODUCTION}

Timing synchronization is crucial for good performance of code division multiple access (CDMA) systems. In this kind of system, timing synchronization is usually divided in two parts. The first one is a coarse technique, which should provide timing synchronization within a period of a chip. In the following, the fine timing stage is responsible to acquire and track the timing with an error much inferior to the chip period. In this paper, we focus our attention in the latter case, i.e., the fine timing stage.

The rationale used in this paper is that a time shift incurs in a phase rotation in the frequency domain. By exploring this property, we have proposed a simple computational noncoherent timing synchronization technique. Its performance is close to the modified Cramér-Rao bound (MCRB) [1] for small roll-off values of the transmitter/receiver filters.

Moreover, the presence of narrowband interference is most likely to occur in broadband systems and in some environments (e.g. power line channels [2]). Such interference can be very strong and its mitigation can offer considerable gain in the performance of the timing estimation. Differently from time domain techniques that were tailored for additive white Gaussian channels (e.g. the early-late gate, Müller \& Muller,

${ }^{*}$ The author thanks Maurice Bellanger for precious insights. etc $[1,3])$, the proposed technique can easily excise such narrowband interferers.

This paper is organized as follows. In section 2 we describe the system model. The timing synchronization technique is introduced in section 3 . Section 4 presents the analytical performance. In section 5 , we assess the performance of the technique with some numerical simulations and compare them to the theoretical results obtained in the previous section and to the MCRB. The computational complexity of the technique is discussed in section 6. Finally, in section 7 , the conclusion and perspectives are stated.

\section{SYSTEM MODEL}

We will consider a multicarrier CDMA (MC-CDMA) system, even though the technique can also be employed to direct sequence CDMA (DS-CDMA) if we use a frequency domain receiver [4].

We take into account only the single user case and a distortionless channel. The analysis under multiuser interference (MUI) is beyond the scope of this paper. However, it is worth noting that MUI can be mitigated by parallel or successive interference cancellation [5] in conjunction with the technique presented herein.

Let us define the $m$-th spread symbol in frequency domain as:

$$
X(k, m)=a(m) C(k)
$$

where $a(m)$ is the $m$-th transmitted symbol, $C(k)$ is the $k$-th chip of the spreading sequence and $0 \leq k \leq K-1$ with $K$ being the spreading factor and an even value.

The spread symbol in the time domain is obtained by performing the inverse discrete Fourier transform (IDFT) given by:

$$
\begin{aligned}
& x(i+m K)=\frac{1}{K} \sum_{k=0}^{K-1} X(k, m) \mathrm{e}^{j 2 \pi k i / K} \\
& x(i+m K)=a(m) c(i), 0 \leq i \leq K-1
\end{aligned}
$$

where $c(i)$ is the IDFT of $C(k)$, and thus the relationship with the DS-CDMA. 
We then insert the cyclic prefix (CP), which is a copy of the last $N_{C P}$ chips of each spread symbol and defining $N_{T}=$ $N_{C P}+K$, the signal in time domain becomes:

$x_{C P}(i+m K)=x\left(K\left\lfloor\frac{i+m K}{N_{T}}\right\rfloor+\left[[i+m K]_{N_{T}}-N_{C P}\right]_{N}\right)$

where $[x]_{y}$ means the modulus function $\bmod (x, y)$.

Finally, the transmitted signal $s(t)$ is the result of the convolution of (3) with a root-raised cosine $g(t)$ that has a roll-off $\alpha$ and a delay $\tau$ representing the propagation delay:

$s(t-\tau)=\sum_{i+m K=-\infty}^{\infty} x_{C P}(i+m K) g(t-(i+m K) T-\tau)$

where $T=\frac{K}{N_{T}} T_{c}$ and $T_{c}$ is the chip period.

The received signal is given by:

$$
r(t)=s(t-\tau)+n(t)
$$

where $n(t)$ is the Gaussian noise with variance $\sigma_{n}^{2}$.

The received signal is convolved with a root-raised cosine $g(t+\hat{\tau})$ with a roll-off $\alpha$ and the estimated delay $\hat{\tau}$ that is used to track the delay $\tau$. Let us define $h(t, \Delta \tau)=g(t-\tau) *$ $g(t+\hat{\tau})=g(t) * g(t-\Delta \tau)$ with $\Delta \tau=\tau-\hat{\tau}$ as being the timing offset. The signal is then sampled at the rate $T$ and the cyclic prefix is removed. Taking the discrete Fourier transform (DFT) of the resulting signal, we have:

$$
R(k, m, \Delta \tau)=a(m) C(k) H(k, \Delta \tau)+N(k, m)
$$

where $H(k, \Delta \tau)=\left(F(k) \mathrm{e}^{-j 2 \pi k \frac{\Delta \tau}{K T}}+F(K-k) \mathrm{e}^{j 2 \pi(K-k) \frac{\Delta \tau}{K T}}\right)$, $F(k)$ is the partial frequency raised-cosine response given by:

$F(k)= \begin{cases}1, & 0 \leq k \leq\left\lfloor\frac{K(1-\alpha)}{2}\right\rfloor \\ \frac{1}{2}\left\{1+\cos \left[\frac{\pi}{K \alpha}\left(k-\frac{K(1-\alpha)}{2}\right)\right]\right\}, & \left\lceil\frac{K(1-\alpha)}{2}\right\rceil \leq k \leq\left\lfloor\frac{K(1+\alpha)}{2}\right\rfloor \\ 0, & \left\lceil\frac{K(1+\alpha)}{2}\right\rceil \leq k \leq K-1\end{cases}$

and $N(k, m)$ is the Gaussian noise in the frequency domain.

By defining $U(k, m, \Delta \tau)=R(k, m, \Delta \tau) C^{*}(k)=$ $a(m)|C(k)|^{2} H(k, \Delta \tau)+N(k, m) C^{*}(k)$, the despreading is achieved by:

$$
\hat{a}(m)=\sum_{k=0}^{K-1} U(k, m, \Delta \tau)
$$

\section{THE FREQUENCY DOMAIN TIMING TECHNIQUE}

We suppose that the coarse timing stage has acquired synchronism within an error smaller than the chip period. Afterward, the fine stage refines the timing estimation and tracks any variation. Many methods for timing synchronization can be used, such as the classic early-late gate, which underlying idea can be performed in the frequency domain [6]. In

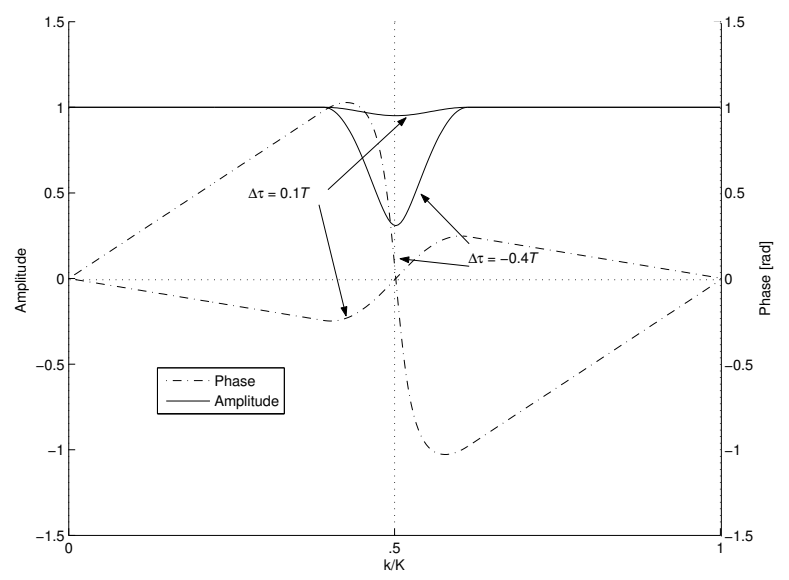

Fig. 1. Frequency response for two different timing offset values and roll-off $\alpha=0.22$

this paper, we propose a simple computational frequency domain technique which can almost attain the MCRB for small roll-off values and has no self-noise. It can also make a direct use of the receiver equalizer, e.g. the matched filter, to excise narrowband interference that may be present in the received signal. In order to demonstrate our technique, let us suppose a simple case where $a(m)=1$ for every value of $m$ and absence of noise. In such a case, we can write $U(k, m, \Delta \tau)=|C(k)|^{2} H(k, \Delta \tau)$. Since $|C(k)|^{2}$ is real and positive, let us analyze $H(k, \Delta \tau)$ for two timing offset, represented in Figure 1. It is easily seen that the phase of $H(k, \Delta \tau)$ is proportional to the timing offset and therefore can be used to estimate it. However, in order to obtain a non-coherent timing estimator, there are two similar problems that must be treated: the symbol and channel phases. The solution to these problems consists in two parts. First, let us suppose that we have a real received sequence $s(t)$. This hypothesis supposes that $a(m)$, IDFT $\{C(k)\}$ and $n(t)$ are real and that the channel has phase 0 or $\pi$. In such a case, independently of the real value assumed by $a(m)$, the phase that corresponds to the delay $\tau$ is proportional to:

$$
\hat{\tau} \propto \arctan \left\{\frac{\sum_{k=1}^{K / 2-1} \operatorname{Im}\{U(k, m, \Delta \tau)\}}{\sum_{k=0}^{K / 2} \operatorname{Re}\{U(k, m, \Delta \tau)\}}\right\}
$$

It is worth noting that we use only half of the DFT because the other half is just the complex conjugate of the first half, with exception of the terms $k=0$ and $k=K / 2$, since the time signal is real valued.

Aiming a less computational intensive approach, i.e., no division or arctan operation, we use:

$$
\hat{\tau} \propto \sum_{k=0}^{K / 2} \operatorname{Re}\{U(k, m, \Delta \tau)\} \sum_{k=1}^{K / 2-1} \operatorname{Im}\{U(k, m, \Delta \tau)\}
$$

Then, in order to make the previous hypothesis true, we can see the received sequence $s(t)$ as the result of the sum of a real and an imaginary valued message and process them as 
real valued signals. By processing each sequence (real part and imaginary part of $s(t)$ ) with its equivalent decomposition of the spreading sequence $c(i)$, which is the IDFT of the sequence $C(k)$, we can apply the estimator (9) in each sequence. Moreover, we can adopt a complex representation of the signal $R(k, m, \Delta \tau)$ and $U(k)=R(k, m, \Delta \tau) C^{*}(k)$ if we make use of the well known property of the DFT which states:

$$
\begin{array}{ll}
\operatorname{DFT}\{\operatorname{Re}\{g(i)\}\} & =\frac{1}{2}\left(G[k]_{K}+G^{*}[-k]_{K}\right) \\
\operatorname{DFT}\{j \operatorname{Im}\{g(i)\}\} & =\frac{1}{2}\left(G[k]_{K}-G^{*}[-k]_{K}\right)
\end{array}
$$

By substituting the above property on (9), the non-coherent timing estimator $e(m)$ is written as:

$$
\begin{array}{ll}
A(k, m)= & U\left([k]_{K}, m, \Delta \tau\right)+U^{*}\left([-k]_{K}, m, \Delta \tau\right) \\
B(k, m)= & -j U\left([k]_{K}, m, \Delta \tau\right)+j U^{*}\left([-k]_{K}, m, \Delta \tau\right) \\
e(m)=\quad & -\sum_{k=0}^{K / 2} \operatorname{Re}\{A(k, m)\} \sum_{k=1}^{K / 2-1} \operatorname{Im}\{A(k, m)\} \\
& -\sum_{k=0}^{K / 2} \operatorname{Re}\{B(k, m)\} \sum_{k=1}^{K / 2-1} \operatorname{Im}\{B(k, m)\}
\end{array}
$$

Finally, the error is integrated in order to estimate the delay $\tau$ :

$$
\hat{\tau}(m+1)=\hat{\tau}(m)+\mu e(m)
$$

where $\mu$ is the adaptation step-size.

\subsection{Improving the estimator performance}

For small values of $\Delta \tau$, the real part of $H(k, \Delta \tau)$ is almost constant for every value of $k$. Nonetheless, the imaginary part varies as a function of $k$. The absolute value of the imaginary part grows as $k$ rises until we fall in the region of aliasing of the raised-cosine filter, where it decreases. The larger the imaginary value, the larger the contribution to the timing offset estimation. Therefore, if we use this knowledge by applying a maximum ratio combining to the imaginary part of the estimator, we can gain some robustness against noise.

The weighting coefficients are obtained for a small value of $\Delta \tau$, since the estimator will be oscillating around $\Delta \tau=0$. We have chosen $\Delta \tau=0.1 T$. A smaller value has practically no additional gain, since the correspondent values of $W(k)$ for each value of $\Delta \tau$ are directly proportional to $\Delta \tau$ when $\Delta \tau / T$ is small.

Let us define the gain that is used to ponder the imaginary part as:

$$
W(k)=|\operatorname{Im}\{H(k, 0.1)\}|, 1 \leq k \leq K / 2-1
$$

Thus, with $A(k, m)$ and $B(k, m)$ as in equation (11), the enhanced estimator $e_{W}(m)$ is given by:

$$
\begin{aligned}
e_{W}(m)= & -\sum_{k=0}^{K / 2} \operatorname{Re}\{A(k, m)\} \sum_{k=1}^{K / 2-1} W(k) \operatorname{Im}\{A(k, m)\} \\
& -\sum_{k=0}^{K / 2} \operatorname{Re}\{B(k, m)\} \sum_{k=1}^{K / 2-1} W(k) \operatorname{Im}\{B(k, m)\}
\end{aligned}
$$

\section{THEORETICAL PERFORMANCE ANALYSIS}

We are interested in the normalized variance of the estimator for its operation point, i.e., around $\Delta \tau=0$. From [1], the normalized variance $\sigma^{2}$ is given by:

$\sigma^{2}=\frac{2 B_{L} T}{S^{2}} \frac{1}{T^{2}} \sum_{m=-\infty}^{+\infty} \mathrm{E}\{e(i) e(i-m) \mid \Delta \tau=0\}(1-\mu S)^{|m|}$

where $S=\left.\frac{\partial\{\mathrm{E}\{e(m)\}\}}{\partial \Delta \tau}\right|_{\Delta \tau=0}$ and the normalized loop bandwidth $B_{L} T=\frac{\mu S}{2(2-\mu S)}$.

For $\Delta \tau=0$, absence of multipath and additive white Gaussian noise, $\mathrm{E}\{e(i) e(i-m) \mid \Delta \tau=0\}=0$ for every $m \neq 0$. Therefore, equation (15) becomes:

$$
\sigma^{2}=\frac{2 B_{L} T}{S^{2}} \frac{1}{T^{2}} \mathrm{E}\left\{|e(m)|^{2} \mid \Delta \tau=0\right\}
$$

Due to the non-coherent nature of the estimator, in order to simplify the calculations we can assume without loss generality that $a(m)= \pm 1$ with equal probability. In such a case, after tedious calculation, we obtain the general formula for any chosen code. But first, let us define the value of $\mathrm{E}\left\{|e(m)|^{2} \mid \Delta \tau=0\right\}$ :

$$
\mathrm{E}\left\{|e(m)|^{2} \mid \Delta \tau=0\right\}=\mathcal{A B}+\mathcal{C D}
$$

where:

$$
\begin{aligned}
\mathcal{A}= & \sigma_{a}^{2}\left(\sum_{k=0}^{K / 2}|C(k)|^{2}+\left|C\left([-k]_{K}\right)\right|^{2}\right)^{2} \\
& +\frac{K \sigma_{n}^{2}}{2}\left(4|C(0)|^{2}+4|C(K / 2)|^{2}+\sum_{k=1}^{K / 2-1}|C(k)|^{2}+\left|C\left([-k]_{K}\right)\right|^{2}\right) \\
\mathcal{B}= & \frac{K \sigma_{n}^{2}}{2}\left(\sum_{k=1}^{K / 2-1}|C(k)|^{2}+\left|C\left([-k]_{K}\right)\right|^{2}\right) \\
\mathcal{C}= & \frac{K \sigma_{n}^{2}}{2}\left(4|C(0)|^{2}+4|C(K / 2)|^{2}+\sum_{k=1}^{K / 2-1}|C(k)|^{2}+\left|C\left([-k]_{K}\right)\right|^{2}\right) \\
\mathcal{D}= & \sigma_{a}^{2}\left(\sum_{k=0}^{K / 2}|C(k)|^{2}+\left|C\left([-k]_{K}\right)\right|^{2}\right)^{2} \\
& +\frac{K \sigma_{n}^{2}}{2}\left(\sum_{k=1}^{K / 2-1}|C(k)|^{2}+\left|C\left([-k]_{K}\right)\right|^{2}\right)
\end{aligned}
$$

$\sigma_{n}^{2}=\frac{1}{2 E_{s} / N_{o}}$ with $E_{s} / N_{o}$ being the symbol to noise ratio. Then, the value of the slope $S=\left.\frac{\partial \mathrm{E}\{e(m)\}}{\partial \Delta \tau}\right|_{\Delta \tau=0}$ is:

$$
\begin{aligned}
& S=\quad-\sigma_{a}^{2} \sum_{k=0}^{K / 2}\left\{\left|C\left([k]_{K}\right)\right|^{2}+\left|C\left([-k]_{K}\right)\right|^{2}\right\} \\
& \times \times \sum_{k=1}^{K / 2-1} \operatorname{Im}\left\{\left.\left|C\left([k]_{K}\right)\right|^{2} \frac{\partial H\left([k]_{K}, \Delta \tau\right)}{\partial \tau \tau}\right|_{\Delta \tau=0}\right. \\
&\left.+\left.\left|C\left([-k]_{K}\right)\right|^{2} \frac{\partial H^{*}\left([-k]_{K}, \Delta \tau\right)}{\partial \Delta \tau}\right|_{\Delta \tau=0}\right\} \\
&-\sigma_{a}^{2} \sum_{k=1}^{K / 2-1}\left\{-\left|C\left([k]_{K}\right)\right|^{2}+\left|C\left([-k]_{K}\right)\right|^{2}\right\} \\
& \times \sum_{k=1}^{K / 2-1} \operatorname{Re}\left\{-\left.j\left|C\left([k]_{K}\right)\right|^{2} \frac{\partial H\left([k]_{K}, \Delta \tau\right)}{\partial \Delta \tau}\right|_{\Delta \tau=0}\right. \\
&\left.+\left.j\left|C\left([-k]_{K}\right)\right|^{2} \frac{\partial H^{*}\left([-k]_{K}, \Delta \tau\right)}{\partial \Delta \tau}\right|_{\Delta \tau=0}\right\}
\end{aligned}
$$

and $\left.\frac{\partial H(k, \Delta \tau)}{\partial \Delta \tau}\right|_{\Delta \tau=0}=-j 2 \pi \frac{k}{K T} F(k)+j 2 \pi \frac{K-k}{K T} F(K-k)$ 
Hence, we obtain the theoretical performance of the proposed technique by replacing equations (17) and (18) on (16).

The theoretical performance of the enhanced technique is obtained by changing the terms $\mathcal{B}, \mathcal{D}$ and the slope $S$ to include the weighting by $W(k)$, defined in equation (13). These terms for the enhanced technique are given by:

$$
\begin{aligned}
\mathcal{B}= & \frac{K \sigma_{n}^{2}}{2} \sum_{k=1}^{K / 2-1} W^{2}(k)\left\{|C(k)|^{2}+\left|C\left([-k]_{K}\right)\right|^{2}\right\} \\
\mathcal{D}= & \sigma_{a}^{2}\left(\sum_{k=0}^{K / 2} W(k)\left\{|C(k)|^{2}+\left|C\left([-k]_{K}\right)\right|^{2}\right\}\right)^{2} \\
& +\frac{K \sigma_{n}^{2}}{2}\left(\sum_{k=1}^{K / 2-1} W^{2}(k)\left\{|C(k)|^{2}+\left|C\left([-k]_{K}\right)\right|^{2}\right\}\right) \\
S=\quad & -\sigma_{a}^{2} \sum_{k=0}^{K / 2}\left\{\left|C\left([k]_{K}\right)\right|^{2}+\left|C\left([-k]_{K}\right)\right|^{2}\right\} \\
& \times \sum_{k=1}^{K / 2-1} \operatorname{Im}\left\{\left.W(k)\left|C\left([k]_{K}\right)\right|^{2} \frac{\partial H\left([k]_{K}, \Delta \tau\right)}{\partial \Delta \tau}\right|_{\Delta \tau=0}\right. \\
& -\sigma_{a}^{2} \sum_{k=1}^{K / 2-1}\left\{-W(k)\left\{\left|C\left([k]_{K}\right)\right|^{2}+\left|C\left([-k]_{K}\right)\right|^{2}\right\}\right\} \\
& \times \sum_{k=1}^{K / 2-1} \operatorname{Re}\left\{-\left.j\left|C\left([k]_{K}\right)\right|^{2} \frac{\partial H\left([k]_{K}, \Delta \tau\right)}{\partial \Delta \tau}\right|_{\Delta \tau=0}\right. \\
& \left.+\left.j\left|C\left([-k]_{K}\right)\right|^{2} \frac{\partial H^{*}\left([-k]_{K}, \Delta \tau\right)}{\partial \Delta \tau}\right|_{\Delta \tau=0}\right\}
\end{aligned}
$$

It can be already observed that when $\sigma_{n}^{2}$ tends to zero the estimator variance of the proposed technique tends also to zero. Therefore, in comparison to the time domain techniques such as the early-late gate and Gardner detector [1], the proposed technique does not suffer from self-noise. This can be explained by the fact that this technique works with the signal sampled at the Nyquist rate. Hence, when there is no timing offset, there is no interchip or intersymbol interference that are in the origin of the self-noise.

\section{SIMULATION RESULTS}

For all simulations, we use QPSK modulation. The estimator variance is obtained by the average of the variance for 35 independent runs, each one with 1600 symbols. We also use, for each run, a random channel phase uniformly distributed between 0 and $2 \pi$. The transmitter/receiver filter was implemented with an oversampling equal to 8 and length $[-7 T, 7 T]$. The timing correction was done by reconstructing the receiver filter taking into account the estimated delay $\hat{\tau}$. The spreading code $C(k)$ used in the simulations was obtained by a pseudo-random sequence generated by the polynomial $p(x)=1+x+x^{6}$ initialized with $\left[\begin{array}{llllll}1 & 0 & 0 & 0 & 0 & 0\end{array}\right]$. To this sequence of 63 chips, it was appended one chip equal to -1 , totalizing $K=64$ chips. It must be noted that other codes with $|C(k)|=1$ for every $k$ would give the same performance. We have effectuated simulations with cyclic prefix sizes $N_{C P}=0,4$ and 16 . However, since the channel has no multipath, the only effect that the cyclic prefix has is a small performance degradation due to the augmentation of the noise variance $\sigma_{n}^{2}$, which is proportional to $N_{T} / K$ for a given $E_{s} / N_{o}$ value. We have considered $N_{C P}=0$ for all simulations shown in this paper.

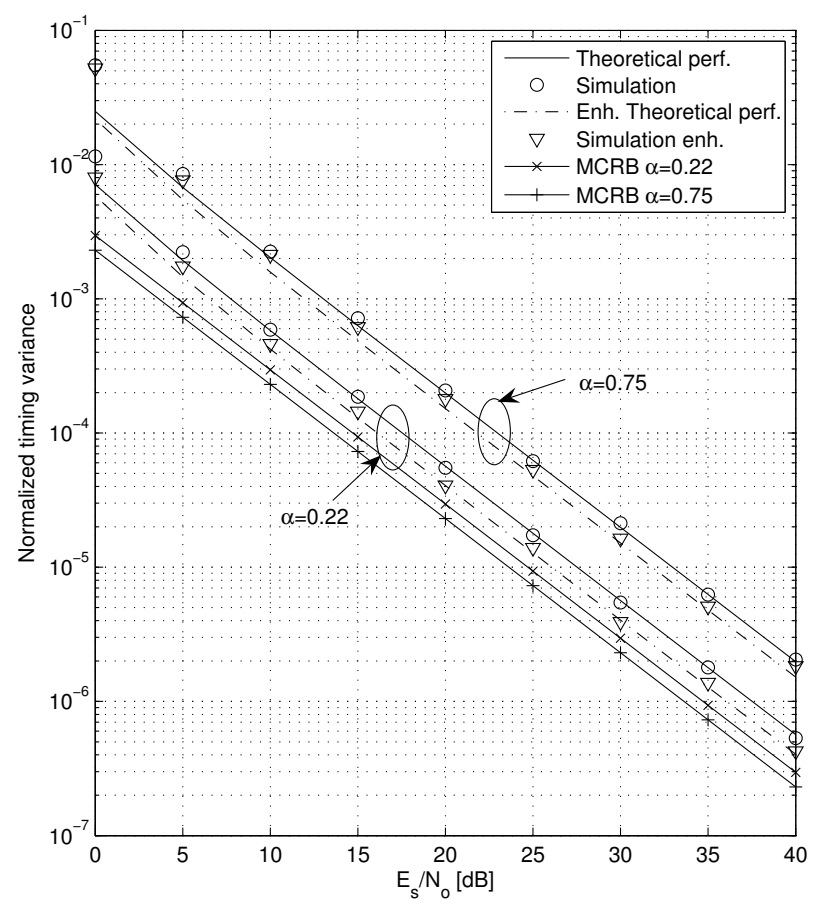

Fig. 2. Normalized timing variance for $B_{L} T=0.01$ and additive white Gaussian noise

We first analyze the performance of the proposed estimator for an AWGN channel. Figure 2 shows the normalized timing variance for the roll-off factors 0.22 and 0.75 . As we can see, the simulation matches the predicted theoretical performance of the estimator, except for very low $E_{S} / N_{o}$. Such behavior may be explained by the larger fluctuations of the estimated delay $\hat{\tau}$, which creates more noise than predicted by the theoretical performance analysis. Furthermore, the performance of the proposed technique compared to the MCRB decreases when the roll-off becomes large. This behavior is similar to the Müller \& Muller time-domain technique that also works at Nyquist sample rate [1]. Finally, the enhanced technique can provide more than $1 \mathrm{~dB}$ when compared to the normal one. It is worth noting that when $\alpha$ tends to zero, the difference of the proposed technique to the MCRB is equal to $1.5 \mathrm{~dB}$ and to the enhanced one is less than $0.1 \mathrm{~dB}$. The simulation results also corroborate with the absence of self-noise.

One considerable advantage of tracking the timing in the frequency domain is the possibility to easily excise narrowband interference. This can be accomplished by applying the channel matched filter to the received signal. In addition, we can use a more pragmatic approach where we simply ignore the subcarriers affected by the narrowband interference. In order to illustrate the gains that can be achieved in this case, we simulate a case with three narrowband interferers. These interferers are generated by filtering white Gaussian noise with an inverse notch filter with poles at $0.99 \mathrm{e}^{j \frac{10 \pi}{64}}, 0.99 \mathrm{e}^{j \frac{56 \pi}{64}}$ and $0.99 \mathrm{e}^{j \frac{90 \pi}{64}}$ and zeros at $0.85 \mathrm{e}^{j \frac{10 \pi}{64}}, 0.85 \mathrm{e}^{j \frac{56 \pi}{64}}$ and $0.85 \mathrm{e}^{j \frac{90 \pi}{64}}$. As illustrated in Figure 3, the narrowband interferers are 24dB stronger than the additive white Gaussian noise. Figure 4 shows the normalized noise variance for this case. 


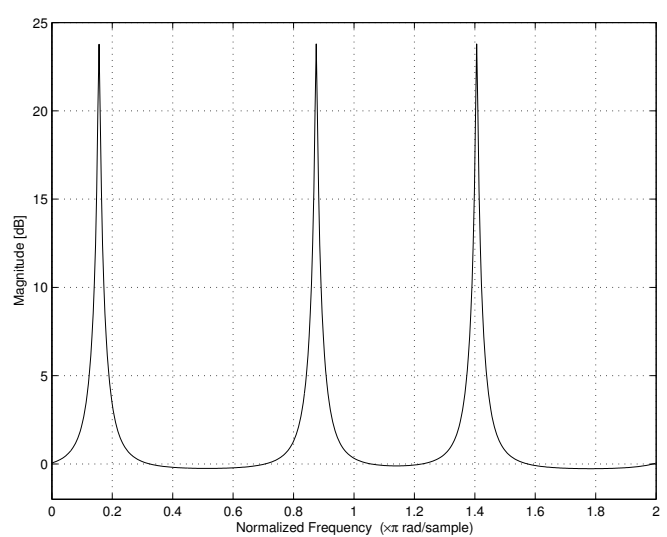

Fig. 3. Power spectrum of the narrowband additive Gaussian noise

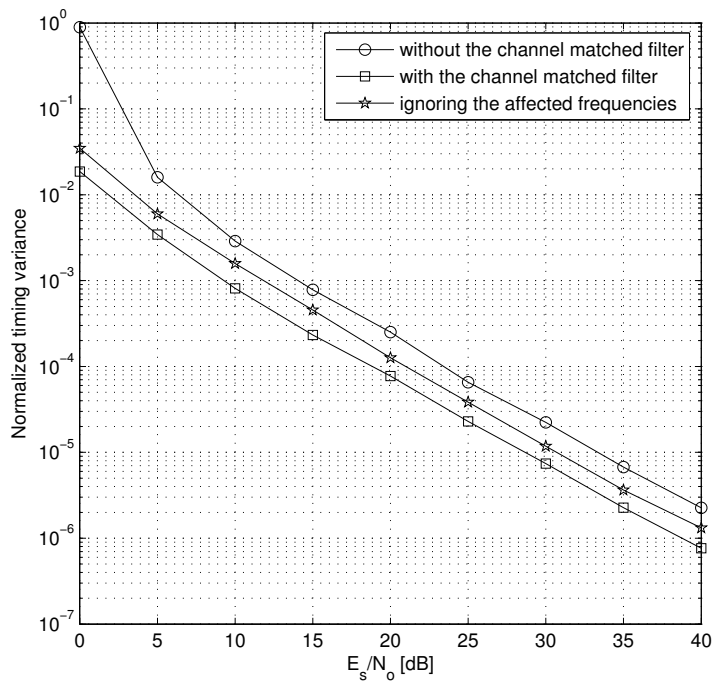

Fig. 4. Normalized timing variance for $B_{L} T=0.01$, roll-off $\alpha=0.22$ and narrowband additive Gaussian noise

We found out that the excision generated by the matched filter can provide a gain larger than $4.9 \mathrm{~dB}$. When we just ignore the subcarriers affected by the narrowband noise, i.e. the subcarriers 5, 28, 45 and their left and right neighbors, the gain is equal to $2.5 \mathrm{~dB}$.

\section{COMPUTATIONAL COST}

The proposed technique is quite simple. Since the despreading of the transmitted symbol, i.e., the $K$ complex multiplication of $R(k, m)$ by $C^{*}(k)$ is obligatory, the complexity of the proposed technique is reduced to 2 real multiplications and $K+2$ complex summations. The enhanced technique needs additional $K-2$ real multiplications. For comparison, an equivalent frequency domain early-late gate technique proposed in [6] for OFDM but that can be adapted for CDMA needs $2 K+2$ complex multiplications and $K$ real summations.

The rejection of the narrowband noise has virtually zero computational complexity cost, since the proposed timing technique can use the equalized signal obtained, in the context of this paper, by the channel matched filter.

\section{CONCLUSION AND PERSPECTIVES}

In this paper we have presented a non-coherent timing synchronization technique in the frequency domain for CDMA systems. It is a simple computational technique that approaches the modified Cramér-Rao bound when the roll-off of the transmitter/receiver filter tends to zero. Due to its operation in the frequency domain, it can easily excise narrowband noise without additional cost, since it can use the equalized signal used for symbol reception.

The technique proposed here can be adapted to a decisiondirected or data-aided joint phase and timing estimation mode. It must also be noted that it can be used with orthogonal frequency division multiplexing (OFDM) systems, where we can use pilot subcarriers for non-coherent timing or joint phase and timing estimation and/or all the data subcarriers in a decision-directed joint phase and timing estimation mode.

\section{REFERENCES}

[1] U. Mengali and A. N. D'Andrea, Synchronization Techniques for Digital Receivers, New York and London : Plenum Press, 1st edition, 1997.

[2] M. Gotz, M. Rapp, and K. Dostert, "Power line channel characteristics and their effect on communication design," IEEE Communications Magazine, April 2004.

[3] H. Meyr, M. Moeneclaey, and S. A. Fechtel, Digital Communication Receivers : Synchronisation, Channel Estimation and Signal Processing, Wiley Series in Telecommunications and Signal Processing, 1st edition, 1997.

[4] C. M. Panazio and M. Bellanger, "On the Frequency Domain Approach for Spread Spectrum Receivers: Towards a Convergence of DS-CDMA, MC-CDMA and OFDM," in IEEE SPAWC, Lisbon, Portugal, July 2004.

[5] U. Madhow and M. L. Honig, "MMSE Interference Suppresion for Direct-Sequence Spread Spectrum CDMA," IEEE Transaction on Communications, vol. 42, no. 12, pp. 3178-3188, December 1994.

[6] Baoguo Yang, K. B. Letaief, R. S. Chengand, and Zhigang Cao, "Timing Recovery for OFDM Transmission," IEEE Journal on Selected Areas in Communications, vol. 18 , no. 11, pp. 2278-2291, November 2000. 